IRSH 62 (20I7), pp. 48 I-494 doi: I0.10I7/S00208590 I 70004 I 4

(C) 2017 Internationaal Instituut voor Sociale Geschiedenis

\title{
The Beginnings of a World History of Modern Migration: The Lucassens's Volume on Globalising Migration History
}

\author{
PATRICK MANNING \\ Department of History, University of Pittsburgh \\ 3904 Posvar Hall, Pittsburgh, PA I 5260, USA \\ E-mail: pmanning@pitt.edu
}

\section{EURASIAN MIGRATION SINCE 1500: RECENT ANALYTICAL ADVANCES}

Jan and Leo Lucassen have assembled an impressive analytical summary of migration across Eurasia from i 500 to the present. In setting their framework, they are especially critical of the state-centered approach of earlier work. That is, they show how national and imperial governments have chosen to narrow their vision of migration to individuals crossing national and imperial boundaries, thus neglecting the many migrants who have moved to distant rural areas, to cities, and the many others who have moved seasonally among such locations. Perhaps more surprising, governments have neglected to include the numerous soldiers and sailors in their own employ within the totals of migrants. Historians of migration, attracted especially by the allure of national comparisons and contrasts, tended for some time to follow official statistics and focus their understanding of migration on the international and especially transoceanic movement of migrants.

In leading an energetic campaign to explore modern-era migration worldwide, the Lucassens are showing that patterns of migration display remarkable parallels among world regions and that the regional specificities, while they definitely exist, are not necessarily those highlighted in the previous literature on migration. Perhaps the most basic point their work underscores is that migration was mostly domestic rather than international. For Europe, they calculate that international migration (immigration and emigration) accounted for a total of thirty per cent of all migration in the period I $85 \mathrm{I}-1900$, but normally remained below ten per cent of all migration; for China, international migration peaked at just over fifteen per cent of total migration in the same period. ${ }^{{ }}$

I. Jan Lucassen and Leo Lucassen, "Measuring and Quantifying Cross-Cultural Migrations: An Introduction", in Jan Lucassen and Leo Lucassen (eds), Globalising Migration History: The Eurasian Experience (I6th-2 Ist Centuries) (Leiden, 2014), pp. I7, 31 . 
These quantitative comparisons of migration over time and space have become possible because of the Lucassens's systematic calculation of cross-cultural migration rates (CCMRs), where each of these rates is reported for a given region over half-century periods. For instance, the steady increase in rates of migration in Europe (including Russia) is estimated to have caused the CCMR to increase from nine per cent (I $50 \mathrm{I}-\mathrm{I} 550$ ) to sixty-six per cent (I85I-I 900). The steady rise in migration rates for Europe plus Russia as a whole is of interest. Of equal interest is the fact that the migration rates for Russia alone, when separated out, appear to be quite parallel in their growth to the rates for the rest of Europe. China and Japan had consistently lower rates of migration roughly half those of Europe - until the late twentieth century, when it seemed migration rates throughout Eurasia were converging to a common level. As the editors state, there was "a common start, a long divergence and a recent convergence" in Eurasian rates of cross-cultural migration. ${ }^{2}$

The CCMR calculation is a new one. Its basic logic - rates of migration for a home population - is clear, but the precise meaning of the CCMR percentage is still in discussion. The CCMR is calculated, for a given region, as the total number of persons who have ever migrated in or from that region in a given period, divided by the population of the region at the midpoint of that period. Some of the fine points of the definitions and calculation of this measure are discussed below. The basic point is that the CCMR calculations, so long as they are performed according to systematic procedures, give results that provide instructive and verifiable comparisons.

Comparisons among CCMRs are already showing Eurasia-wide parallels in migration patterns, as mentioned above, but also show instances of distinctiveness. The rates of migration reported in this volume for China and Japan are surely higher than most scholars will have expected, especially before the mid-nineteenth century. The high and rising migration rates for Russia and China, but also for Japan, will be challenging to those who have previously assumed that intensive migration was a property of North Atlantic societies and no others. Other distinctive results are the shifts in city populations, notably the widespread decline in urbanization during the eighteenth century, and the nineteenth-century migrations to tropical plantations in Asia.

This volume on Eurasian migration is to be followed by parallel studies of other world regions from the same editors, and it is not too early to suggest that the Eurasian patterns - here revealed in broad detail as never before will turn out to be representative of other world regions as well. One must 
note that not all Eurasian regions have yet been documented in enough detail to propose CCMRs for them. The chapters on South Asia and Southeast Asia reflect work that is at an earlier stage of development, especially because the underlying documentation is far weaker than for Europe and northern Asia; one expects that CCMRs will result from further study. Southwest Asia, similarly, requires further analysis, and it is being conducted in a project that includes African migration. But I think that the initial results reported in this volume are robust enough to give a clear idea as to the range of migration rates for all areas of the world in the era since $\mathrm{I} 500$. That is, we may expect that migration rates will prove to have been relatively high for sparsely populated regions, such as the Americas, the Pacific, and Southeast Asia. In contrast, migration rates will probably turn out to be relatively low for densely populated South Asia, and perhaps also for sub-Saharan Africa, where population has been moderately dense. ${ }^{3}$ In such a comparison, densely populated Europe may end up appearing as a region where migration was surprisingly high.

This book, therefore, brings to the reader the beginnings of a world history of migration in the modern era, showing the transformations among the various types and directions of migration, and opening the door to a serious review of the significance of migration in multiple dimensions of social and cultural change. There will be much to debate as this expanded perspective on modern history comes to light. As an instance of a point that might be discussed, the Lucassens begin their book with a clear attempt to escape the constraints that states have placed on the definition, documentation, and understanding of migration. Yet, their analysis returns again and again to states: that is, to the role of empires in migration and the roles of migration in empire. This analysis will be revealing but, in addition, it will be important to explore in detail the importance of migration for regions that have been outside the control of large states.

\section{PROPOSED FINE-TUNING OF THE FRAMEWORK}

Now that the system has been launched, the analysis and calculation of CCMRs needs some "fine-tuning", as the editors note. In this section, I raise two sorts of questions about the precise formulation of the CCMR. I definitely have no objection to the basic notion of the CCMR. Indeed, the hope of having a generally acceptable measure of the rate of migration - and

3. It will soon be possible to compare African migration rates after 1650 with those included in this volume, with the completion of a combined set of estimates of population and migration for numerous African regions. Patrick Manning and Scott Nickleach, "African Population, 1650-1950: The Eras of Enslavement and Colonial Rule", forthcoming. 
the possibility that it could be scaled so as to be discussed both at localized and large-scale levels - brings an important advance. That is, if we could compare societies and regions according to their populations and their migration rates over time, this would constitute a major step forward in understanding human history.

Here, I offer some comments on the definitions linked to the crosscultural migration rate, followed by some comments on the calculation of such rates. Firstly, Jan and Leo Lucassen wisely distinguish the term "mobility" (the full range of movement of individuals) from "migration", a defined subset of all movements. They have summarized the development of their definition and calculations in work since 2008.4 In addition, since the editors have generously cited and drawn on my theoretical work, beginning in 2005 , in assembling their definitions and methods for CCMR, I will briefly compare my original formulation to their current method.' My typology and theory were to apply to the earliest migrations of Homo sapiens, yet, also to extend, in general, to the present day. Thus I, too, distinguished mobility and migration, then took the step of defining human communities as groups of people defined by a shared language and set of customs, living in a "habitat" - an ecological zone in which a community can function with a given set of customs. ${ }^{6}$ I then defined four categories of migration: "home-community migration" (movements within the habitat); "colonization" (settlement beyond the home community in new habitats that could nonetheless be exploited with the same customs); "wholecommunity migration" (in which whole communities moved to a new habitat, often seasonally, and often along with the movements or seasonality of a major resource such as fish or game). Finally, in "cross-community migration" I proposed a type of migration that was unique to humans, in that the development of language and culture brought significant differentiation among communities. As a result, in order to join a new community people were required to learn a new language and new culture. In this typology, I sought to follow Dingle's injunction that the analysis of migration should be in "behavioral" terms, emphasizing the social function of each type of migration, rather than in "ecological" terms that focused mainly on the points of origin and destination. ${ }^{7}$

4. Lucassen and Lucassen, "Measuring and Quantifying”, pp. 5-I4.

5. Patrick Manning, "Cross-Community Migration: A Distinctive Human Pattern", Social Evolution E History, 5:2 (2006), pp. 24-54; idem, Migration in World History (London, 2005).

6. By "customs", I mean the full range of language, technology, and social organization. These characteristics varied among human communities to a far greater degree than among communities of any other species, so that migration among human communities provided an important source of exchange and learning. For a discussion of mobility vs. migration among a broad range of biological species, see H. Dingle, Migration: The Biology of Life on the Move (Oxford, 2006), p. Iо.

7. Manning, “Cross-Community Migration”, pp. 26, 30-33; Dingle, Migration, pp. 36-38. 
This requirement of learning, in order to settle into a new community, built learning skills and encouraged steady transformation in skills and identity for all humans associated with such migration. Within cross-community migration, I defined several sub-types of migration, according to their patterns: settlers (who joined a new community permanently), sojourners (who migrated for a time and then returned home), itinerants (who moved from place to place, perhaps practicing a trade), and invaders (who sought to seize control of their new community). I argued that these categories are still sufficient to describe migration today, but noted changes because of the greater scale of today's society. I left my model as conceptual and did not attempt to quantify it with implementation through data on specific historical situations. ${ }^{8}$ In the assumptions, I assumed that migrants in any category might stay at their initial settlement, or return home, or migrate to yet another place.

The Lucassens have revised this framework, partly to fit their additional insights and partly to fit more specifically to the social patterns of the world since I 500. They have left "home-community migration" outside the discussion, much as I did. Where I used the term "cross-community migration", they have chosen to use the term "cross-cultural migration", though the meaning is much the same. Where I had two levels for migration (the home community as opposed to all other communities to which migrants might move), Lucassen and Lucassen have three levels of migration (the home community from which migrants might depart, a larger territory within which migrants might conduct various sorts of migration, and areas beyond the territory to which migrants might emigrate and from which they might immigrate)..$^{9}$ A particular reason for this shift in the Lucassens's definition is that defining a "territory" (usually a national territory) enables them to identify a population for that territory and thereby calculate rates of migration as a proportion of the territorial population. ${ }^{\circ}{ }^{\circ}$ As will be seen, this shift also requires further changes in the definitions.

We have each assumed that migration took place once when one left the home community. Much of the Lucassens's analysis takes place within territorial units, units that encompass cultural boundaries of language and region. Within territorial units, they define four categories of cross-cultural migration: colonization

8. For instance, one could ask whether the subcategories of settlers, sojourners, itinerants, and invaders are properly seen as elements of a behavioral typology, or whether they reflect ecological categories.

9. Lucassen and Lucassen, "Measuring and Quantifying”, pp. I 3-I4; J. Lucassen and L. Lucassen, "The Mobility Transition Revisited, I 500-1900: What the Case of Europe Can Offer to Global History", Journal of Global History, 4:3 (2009), pp. 347-377.

I0. The Lucassens do not use the term "habitat", but by speaking of migration to the city or from one rural area to another as "cross-cultural" even when it remains within the same "territory", they are using the "culture" within "cross-cultural" in much the same way as I used "habitat" and "home community". Lucassen and Lucassen, "Measuring and Quantifying”, p. I4. 
(rural-to-rural migration), migration to cities, seasonal (migration to and from a destination within a year), and temporal multi-annual (temporary migration with return after multiple years in migration). These four types of "crosscultural migration" parallel the four types of "cross-community migration" that I defined (settlers, sojourners, itinerants, invaders). In addition, because they define a territory and its population for the study of migration rates, the Lucassens must also define emigrants (who leave the unit) and immigrants (who enter the unit). For this reason, the Lucassens have two categories, emigration and immigration, for which I have no equivalents; I defined the migrants to be in one of my four categories no matter how far they migrated. The Lucassens define "colonization" slightly differently than I did, using the term to mean rural-to-rural migration within a single national or imperial territory or political unit. This term shares the meaning I used of maintaining the same customs and social organization while migrating. But where I would label migration from Scotland to Nova Scotia as colonization, the Lucassens label it as emigration. ${ }^{\text {II }}$ Another distinction is that two or perhaps four categories in the Lucassens's model appear to assume that migration is permanent: colonization and migration to cities (but maybe also emigrants and immigrants). Return migrants are put into two categories, seasonal or temporal multi-annual, according to whether their return was after more or less than one year.

As for the measurement of migration according to the CCMR method, there are several inherent limits on the precision of any measurement. Nevertheless, if a common agreement can be developed on the best way to estimate rates of migration, the resulting standard measures can be of great help in comparing migratory practices and experiences over time and space. One question is that of the appropriate time frame for measuring migration. In my experience with reviewing data on Atlantic migrations of both free and enslaved persons, I tend to see cycles of roughly thirty years in the rise and decline of migration from a given port of departure to a destination. But these thirty-year waves began at uneven times, rather than neatly at the start of a decade or century. Is there any way to capture the cycles of migration in our interregional comparisons?

Another set of questions about the measurement of migration concerns whether to measure outward migration, return migration, or both ("net migration"). The figures on South Indian migration, as shown in Sunil Amrith's chapter, illustrate this issue clearly. ${ }^{\mathrm{I} 2}$ The number of immigrants to

I I. In sum, while the two typologies are close in meaning and evolving gradually, it may be that the Lucassen typology is rather more "ecological" and the Manning typology more "behavioral". In addition, colonization as I defined it is not cross-cultural migration, in that the colonists maintain their old customs in the new place.

I 2. Sunil S. Amrith, "South Indian Migration, c.1800-1950", in Lucassen and Lucassen (eds), Globalising Migration History, pp. I35-1 38 . 
Ceylon from I 843 to I94I averaged some I I0,000 per year; the maximum number of migrations for Ceylon was the number of arrivals plus the number of departures: an average of some 200,000 per year from I 843 to I94I; the net migration into Ceylon in the same period (immigrants less emigrants) averaged some 20,000 per year. Immigrants, gross migration, net migration - each of these figures can be calculated with precision and each has some relevance, but they differ greatly in their magnitude and their meaning. Migration historians will need to develop assessments of the best ways to use these varying statistics. To note a parallel issue, one could suggest that it would be relevant to track the distance covered by various migrants, or the time they spent in traveling or at their destination.

An equally complicated and perhaps more important issue is that of the meaning of the cross-cultural migration rate, the percentage that is constructed to calculate it, and the adjustment of the CCMR to take account of changing life expectancy. The CCMR has as its denominator the population of the region under study at the midpoint in the period (thus, for the period I $80 \mathrm{I}-\mathrm{I} 850$ it would be the population at the end of I 825 ). The numerator of the CCMR is the number of persons within that region who ever migrated during that period. ${ }^{{ }^{3}}$ But the resulting percentage is not exactly the proportion of the population that migrated: it is the number of migrants for the whole period as a percentage of the population at a single moment. An alternative would be to try to count the number of migrants in the period as a percentage of the number of persons who ever lived in that period. An additional complication has to do with the difference between the number of migrants and the number of migrations: one might wish to give recognition to the experience of those who migrated multiple times in their life, or at least to account for return migrations as well as outward migrations. (The Lucassens eliminated double counts, so that the estimated number of migrations is closer to the number of initial outward migrations.)

As a first step in adjusting for such issues, the Lucassens have added a correction for life expectancy. Shorter life spans in earlier times means that, for a given mid-period population, there were more people who lived in that period. So they have multiplied the population by a factor intended to standardize all the populations at an expectation of life at birth of fifty years. This adjustment effectively raises the population size and lowers the calculated migration rate for earlier times when the expectation of life was lower; similarly, it lowers the population size and raises the migration rate for later times. ${ }^{\mathrm{I}}$

I 3. Note that this a low estimate of migration in that persons who migrate multiple times are intended to be counted only once.

I4. Comparison of the unadjusted Figure 2 and the adjusted Figure 3 shows this adjustment to be significant and therefore valuable (Lucassen and Lucassen, "Measuring and Quantifying", pp. I7, 23). On the other hand, the current linear adjustment might be replaced with one that was more sensitive to lifespan at the young-adult ages that were most common for migrants. 
One further point is that the midpoint population is not the same as the total number of people at risk of becoming migrants in a fifty-year period, so that the CCMR percentages cannot be interpreted strictly as the probability that a person who lived in the given time and place would migrate. For instance, if the migration rates were calculated for a twenty-five-year period for a similar population, they would be roughly half those for a fiftyyear period; if the migration rates were calculated for a one-hundred-year period and a similar population, they would double. To get closer to a calculated rate that reflects the "probability of migration" within a given population, it might be better to use as a denominator the total number of person-years lived and, as a numerator, the total number of person-years spent as a migrant. That would be the proportion of total experience spent as a migrant for any base population within a given time. In this case, the calculation could be carried out for a long or short period but with comparable rates. Of course, the actual calculation of such a migration rate is not easy. It would require data that are difficult to estimate, but it can be considered a conceptual target. Another way to envision this calculation, one that would yield lower rates of migration than the currently calculated CCMRs, would be to estimate the total number of persons who have ever migrated during a period as a proportion of the person-years lived in that period. This estimate would yield the proportion of people in a given base population who ever migrated for a given time period.

As can be seen, at some future stages the CCMR estimates might undergo what the Lucassens have called "fine-tuning", so that CCMR will hopefully become a dependable and consistently calculated historical measure. Even then, our measures will generally fall short of real precision because of the complexity and variety of social issues. Perhaps researchers should develop the practice of indicating, alongside their estimations of rates of migration, a qualitative judgment on whether they are likely to be underestimates or overestimates.

\section{COMMENTS ON SELECTED CHAPTERS}

Almost half of the chapters in the book include efforts to calculate crosscultural migration rates; for Russia i 500-I900 (Kessler), China I600-1900 (McKeown), Japan I600-2000 (Leo Lucassen et al.), and Indonesia I9002000 (Van Lottum). In a helpful comparative graph, Kessler shows CCMRs for Europe, China, and Russia from I 50 I to I 900 : it indicates that Russian CCMRs paralleled those of Europe in each half-century, while Chinese rates fell from two thirds of European rates before 1750 to one third of European rates in the nineteenth century. ${ }^{15}$ Kessler's estimates show that, in

I 5. Gijs Kessler, "Measuring Migration in Russia: A Perspective of Empire, I 500-1900", in Lucassen and Lucassen, Globalising Migration History, p. 83. 
parallel with Europe, Russian migration relied especially on temporal multi-annual migration, declining in proportion over time, as it did in Europe. This raises the interesting question of how it was possible to have such substantial migration and yet have many peasants held in serfdom. Estimates of migration by sub-region within Russia's huge territory, if they could be calculated, would be of great interest.

Adam McKeown prefaces his CCMR estimates for China by arguing that such estimates, while carried out on a worldwide canvas, do not yet provide us with a world history of migration. This system of analysis "does not provide tools to map broad patterns that cross regions, or to perceive large scale similarities and differences that become apparent over long time frames and by comparing large geographic regions". Further, he warns of the danger of "inappropriate projection of assumptions about causes and effects onto other historical migrations without adequate research". Nonetheless, he participates energetically in preparing CCMR estimates for China, arguing that "The CCMR-model offers an excellent point of departure to embark upon a comparative global history of migration". ${ }^{16} \mathrm{In}$ his calculations, McKeown estimates half-century rates of migration by colonization, emigration, urban migration, and military migration, comparing each with European rates. He argues, however, that a broader similarity of patterns in China and Europe appears when one compares the total of colonization and emigration for each region: indeed, these totals were very close to equal until the early nineteenth century, when such European migration rose while that for China fell.

Two more sets of CCMR estimates are offered - for Japan and Indonesia. For Japan, Leo Lucassen, Osamu Saito, and Ryuto Shimada have assembled numerous data on topics such as emigration, urbanization, and military migration, and have developed fifty-year estimates of CCMR from I 600 to 2000 , with figures above eight per cent for the seventeenth century, below three per cent from 1700 to 1850 , and rising rapidly thereafter to over forty per cent (mostly urbanization) after $1950 .{ }^{17}$ Since Japan is a relatively compact and well-documented region, it is possible for the reader to see the mix of data and assumptions in these estimates with relative clarity. In one other set of estimates intended to support CCMR calculations, Jelle van Lottum estimates "migrant stock rates" for twentieth-century Indonesia. In estimates for 1930, 1960, and 2000, he totals migrants for four of the six categories: urbanization, migrants to land, emigration, and immigration and leaving aside seasonal and temporal multi-annual migrants. The results

16. Adam McKeown, "A Different Transition: Human Mobility in China, I600-1900", in Lucassen and Lucassen, Globalising Migration History, p. 279.

17. Leo Lucassen, Osamu Saito, and Ryuto Shimada, "Cross-Cultural Migrations in Japan in a Comparative Perspective, 1600-2000", in Lucassen and Lucassen, Globalising Migration History, pp. 362-409. 
of the estimates give migrant stock rates of four per cent (I930 and I960) and nine per cent (2000), with urbanization accounting for most of the total. As Van Lottum shows clearly, these rates are calculated differently from CCMRs. These rates are calculated for a single moment (or year) rather than a fifty-year period, and they tabulate the stock of migrants of each type at that moment rather than the flow of migrants moving within a fifty-year period. The population is the total population of a region at that moment, which is somewhat comparable to the midpoint population for a period. Depending on the adjustments made to these estimates, and while the two calculations have various differences, I would expect migrant stock rates in general to have been somewhat lower than CCMRs. At a speculative level, however, it is interesting to note that the addition of seasonal migrants and temporal multi-annual migrants to Van Lottum's totals would bring his migrant stock rates for Indonesia to levels not far off Lucassens's estimates of CCMRs for twentieth-century Europe.

Other chapters in the book make no direct attempt to estimate CCMRs. Some show efforts to calculate migration statistics that might eventually be accumulated into CCMR figures, while others emphasize qualitative approaches, sometimes pointing to the limits of quantitative analysis. Ulbe Bosma's study of migration in nineteenth-century Java focuses on domestic migrants but does include data on pilgrims bound for Mecca, while Sunil Amrith's chapter on South India focuses mainly on cross-territorial migration and Ramaswamy's tracing of migrating weavers in South India up to the eighteenth century can be treated as a step in documenting Indian migrations more broadly. ${ }^{18}$ In contrast, Mireille Mazard's approach to the history of migrations by members of the highland-dwelling Lisu ethnicity among the gorges of Southeast Asia's great rivers notes the multiple roles that can be played by any single migrant. For such groups, often beyond state control, Mazard considers "the cultural aspects of migration history" and treats migration as "a flexible cultural strategy", yielding patterns that "do not conform exactly" to the CCMR framework. In other studies, the contributions of Umeno on immigrants in Manchuria (I $850-$ I 93 I) and of Shen on post-World War II migration in China provide welcome data on major migratory patterns. Ota's view of migrations across the greater Malay Archipelago, 1750-1850, emphasizes expanding rates of migration even before the impact of global industrialization. ${ }^{19}$

I 8. Ulbe Bosma, "Migration and Colonial Enterprise in Nineteenth Century Java", in Lucassen and Lucassen, Globalising Migration History, pp. I I-I79; Amrith, "South Indian Migration, c. I800-1950"; Ramaswamy, "Mapping Migrations of South Indian Weavers Before, During and After the Vijayanagar Period: Thirteenth to Eighteenth Centuries”, in Lucassen and Lucassen, Globalising Migration History, pp. 91-I 2 I.

I9. Mireille Mazard, "The Art of (Not) Looking Back: Reconsidering Lisu Migrations and 'Zomia'”, in Lucassen and Lucassen, Globalising Migration History, pp. 2 I 5-246; Yuki Umeno, 


\section{ADDING A LONGER-TERM PERSPECTIVE}

This volume's studies provide data on an impressively long period of migration history for a huge area. The results are extremely valuable for setting into context the migrations of the twenty-first century, which could be expressed in terms of CCMRs without great difficulty. In this section, however, I want to offer some qualitative comments intended to provide an even longer-term context for the five centuries of migration history presented in this volume.

To start at what one might call the beginning, the initial settlement of Eurasia by Homo sapiens took place some 70,000 to 30,000 years ago. In the early parts of that period, humans moved eastward along the Indian Ocean shores, reaching Australia and New Guinea by 50,000 years ago. Areas of settlement are marked by the river-valley homelands of major language groups of today: Dravidian in India, Sino-Tibetan in Burma, Austric in Vietnam. By 40,000 years ago, humans had found their way north to the temperate regions and rapidly occupied lands of Central Asia, Europe, and northeast Asia.

At a time perhaps 25,000 years ago, a concentration of human population formed in or near the lower Amur Valley, and spread in many directions from there. The languages of this group, the Eurasiatic languages, ultimately became dominant in all of northern and western Eurasia. Some migrants moved south to Japan and Korea, others moved north to Chukotka, but most moved to the west: the Mongol speakers to Mongolia, the Uralic speakers to the Arctic, the Altaic speakers to Central Asia, and the Indo-European speakers further west. This westward movement, long ago, provides a remarkable contrast to the recent and much more rapid expansion in the same territory by eastward-moving Russian speakers.

After the end of the Ice Age, agriculture emerged and spread in tropical and temperate areas beginning some 10,000 years ago. The Indo-European speakers took up farming wheat and barley and expanded to dominate all of Europe, later moving east and south to settle in Iran and North India. The development of rice culture in South and Southeast Asia was accompanied by yam cultivation in Southeast Asia and the arrival of sorghum in northern China.

In a different sort of migratory expansion starting roughly 4,000 years ago (2000 BCE), a mix of Indo-European- and Altaic-speaking peoples developed horse-drawn chariots as instruments of war. After fighting among themselves for some time, they turned south and, in short order,

"Han Chinese Immigrants in Manchuria, I $850-193$ I", in ibid., pp. 307-334; Jianfa Shen, "From Mao to the Present: Migration in China since the Second World War", in ibid., pp. 335-36r; Atsushi Ota, "Toward Cities, Seas, and Jungles: Migration in the Malay Archipelago, c. 1750-1850", in ibid., pp. I80-214. 
conquered all the major states of southwestern Asia and Egypt, invaded North India and Iran, and imposed their weaponry if not their administration on China.

The period from roughly $500 \mathrm{BCE}$ to $\mathrm{I} 200 \mathrm{CE}$ brought a succession of major empires along the southern fringe of Eurasia, accompanied by the expansion of widespread literate culture, a commercial network, and the religions of Buddhism, Christianity, Hinduism, Islam, and other faiths. As a result, the interconnections among the various regions of Eurasia grew more common. From the thirteenth century Mongol armies subsumed most of these regions into a single imperial network. Communication across Eurasia expanded not only during the century of Mongol hegemony, and despite the disasters of the Black Plague; it also experienced resurgence with the rise of successor states.

Comparing this long sequence of Eurasian migrations with the period after I 500, it is interesting to note that, despite what must be the expanded volume and rate of migration since I 500 , the recent displacements have mostly not led to a remaking of the ethnic map. The eastward movement of Russians is one exception and the northeast-northwest movement of Chinese is another, but it might be that earlier migrations were more effective in reshaping Eurasian ethnicity and culture than those of recent centuries.

THE GROWING ROLE OF DIASPORA IN SOCIETY

Lucassen and Lucassen emphasize how major historical debates on economic growth, state formation, and sociocultural change have been pursued with little attention to migration. ${ }^{20}$ In their concluding chapter, the editors argue counter to the implicit assumption that migration has been of marginal importance to major political and socioeconomic changes. In particular, they rely on Charles Tilly's distinction of "capital intensive" and "capitalized coercion" paths of state formation to argue that variations in urbanization, colonization, and temporary multi-annual migration have influenced state formation and the growth of markets. ${ }^{2}{ }^{2}$

As an additional framework for emphasizing the role of migration in the big issues of political and socioeconomic change, I wish to emphasize the growing role of diaspora as a factor in modern history. Especially for recent centuries, in which the rate of migration has increased and in which communication among migrants and with the homeland has improved, the role of diaspora communities has surely become formative in human society.

20. Lucassen and Lucassen, "Measuring and Quantifying", p. 3.

21. Jan Lucassen and Leo Lucassen, "Summary and Concluding Remarks", in Lucassen and Lucassen, Globalising Migration History, pp. 424-428. 
As communications have improved - and as literacy has grown - migrants and their descendants have become increasingly able to maintain contact with their home communities and with distant groups of settlers. Diaspora communities have communicated with their homelands for over two centuries by mail, by remittances of earnings, through newspapers, and by travel - they have thus been better able to sustain their common identity and heritage. Within the past century the mode of travel has come to include aircraft, and communication has become electronic. In each region of settlement, rural or urban, settlers have formed ethnic and regional associations to advance their common concerns in the diaspora and the homeland.

Widely discussed examples of diaspora communities include diasporas from Africa, Italy, India, and China. In addition, given the success of this book in showing that migrant destinations are primarily within their home political unit, it seems appropriate to consider expanding the term "diaspora" to additional migrants. In particular, we can consider labelling as "diasporas" the migrant communities of Russians in Siberia and the Pacific, the Han Chinese settlers in Manchuria and the northwest of China, and the westward-moving movement of numerous communities in the US. ${ }^{22}$

The point of identifying diasporas is not simply to label them but to identify their importance in social change, at both national and transnational levels. One outstanding example is that of Giuseppe Garibaldi, who led a group from the Italian diaspora in South America to land in Italy in 1848 and accelerate the struggle for national unification. Diasporas have not generally had armies or governments, but they have had newspapers, enterprises, and financial contributions that have changed politics and culture in many parts of the world. Gandhi began his political campaigns in the Indian diaspora, the Irish diaspora was central to the achievement of Irish independence, and the Dutch diaspora in Indonesia and the Indonesian diaspora in the Netherlands have transformed both countries.

Analysing diasporas is probably best done at the global level rather than the continental level. Within the confines of Eurasia, however, this volume documents some important diasporas deserving more attention. One such instance is the large Japanese diaspora, military and civilian, throughout the Japanese empire in the I930s and I940s. Similar reasoning, however, can also be effective in considering such small-scale diasporas as that of the Lisu of Southeast Asia. ${ }^{23}$

22. Patrick Manning, The African Diaspora: A History through Culture (New York, 2009); Donna Gabaccia, Italy's Many Diasporas (London, 2000); Lynn Pan, Sons of the Yellow Emperor: A History of the Chinese Diaspora (New York, I994); Lewis H. Siegelbaum and Leslie Page Moch, Broad Is My Native Land: Repertoires and Regimes of Migration in Russia's Twentieth Century (Ithaca, NY, 20I4); David Hackett Fisher and James C. Kelly, Bound Away: Virginia and the Westward Movement (Charlottesville, VA, 2000).

23. Mazard, "The Art of (Not) Looking Back", pp. 2i 5-246; Lucassen et al., "Cross-Cultural Migrations in Japan in a Comparative Perspective”, pp. 387-395. 


\section{QUESTIONS POSED BY THE EDITORS}

In their summary to the volume, the editors raise several questions about the directions in which this study of migration might lead. They ask whether the CCMR method can reasonably be expanded to other parts of the world - and provide evidence that it should be possible, though with diminishing returns for poorly documented regions. As a further point, I would urge that the methods and definitions of CCMR should be discussed and fine-tuned until they represent a commonly accepted variable in social science analysis.

Lucassen and Lucassen ask what sociocultural effects arise from the various types of migration. They wish to know whether urbanization or colonization had greater social influence. As they note, the typology of migration includes numerous sub-types, so that migratory processes could have numerous and even contradictory influences. In particular, they ask about the role of coercion in migration and the consequences of such coercion. Further, they ask about the effects of migration on institutions of the regions of departure and settlement, as well as about the lives of individuals.

Finally, the editors ask: what role should migration play in the debates over global historical patterns, notably the Great Divergence debate? On this last point, it should be underscored that the study of migration provides a steady reminder of how central are interactions of individuals and communities in the lives of migrants and those they meet. Considering this issue helps us not only pay attention to the empirical facts of migration but also to remember that continuous processes of migration have sustained the unity and commonality of human communities. The old models of isolated and distinctive communities, dominant in earlier eras in social science, must be updated to acknowledge this important complication. 